Trauma Berufskrankh 2015 - 17[Suppl 1]:4-9 DOI 10.1007/s10039-013-1991-0

Online publiziert: 7. März 2014

(c) Springer-Verlag Berlin Heidelberg 2014

\section{J. Gabel}

Abteilung für Fußchirurgie, BG-Unfallklinik Murnau

\section{Funktionsanalyse des Fußes}

\section{Anatomische und biomechanische Aspekte für die chirurgische Praxis}

gen Gewölbe des Greiffußes bildete sich durch spiralige Anordnung der knöchernen Elemente ein Spiralgewölbe aus, wobei der erste Strahl mit der Großzehe flach angelegt ist.

Der Fuß ist der komplexeste Skelettabschnitt des Menschen [7] und daher häufiger von Überlastungsschäden, Verletzungen und Krankheitsprozessen betroffen.
Auch sind gewisse bauliche Merkmale wie die kardangelenkähnliche Beweglichkeit des oberen (OSG) und unteren Sprunggelenks (USG) und die Verankerung des ersten Strahls an den Keilbeinen anfällig für Pathologien. So hindert die Sprunggelenkkonstruktion den Menschen nicht nur daran, lange stabil auf einem Bein zu stehen, er ist auch häufig von Umknickchen Entwicklung der Hand zum komplexen Tast- und Greiforgan sowie der Hirnentwicklung charakterisiert.

Lange bevor der Mensch sesshaft wurde, legte er lange Wegstrecken als Nomade zurück. Damit diente der Fuß nicht mehr länger dem Greifen, sondern der Fortbewegung des Zweibeiners Mensch. Der wesentliche Gestaltwandel vollzog sich im Rückfuß, mit Reduktion der ursprünglich 5 auf 2 tarsale Knochen und der Vertikalisierung des Rückfußes durch Verwringung von Kalkaneus (Fersenbein) und Talus (Sprungbein). Dabei kommt zur pronatorischen, nach außen gerichteten $\mathrm{Be}$ wegung des Kalkaneus eine supinatorisch, nach innen gerichtete Gegenbewegung des Talus hinzu. Zusätzlich kam es zu einer massiven Verstärkung und Medialisierung des Tuber calcanei und einer Anhebung des Sustentaculum tali. Dieser auch als Balkon des Fußes bezeichnete Anteil des Fersenbeins trägt mit seiner medialen Gelenkfacette im subtalaren Gelenk wesentlich zur Stabilisierung des Talus auf dem Kalkaneus bei.

Auch der Mittel- und Vorfuß passten sich der Funktion als Trag- und Fortbewegungsorgan an. Aus dem halbkugelförmi-

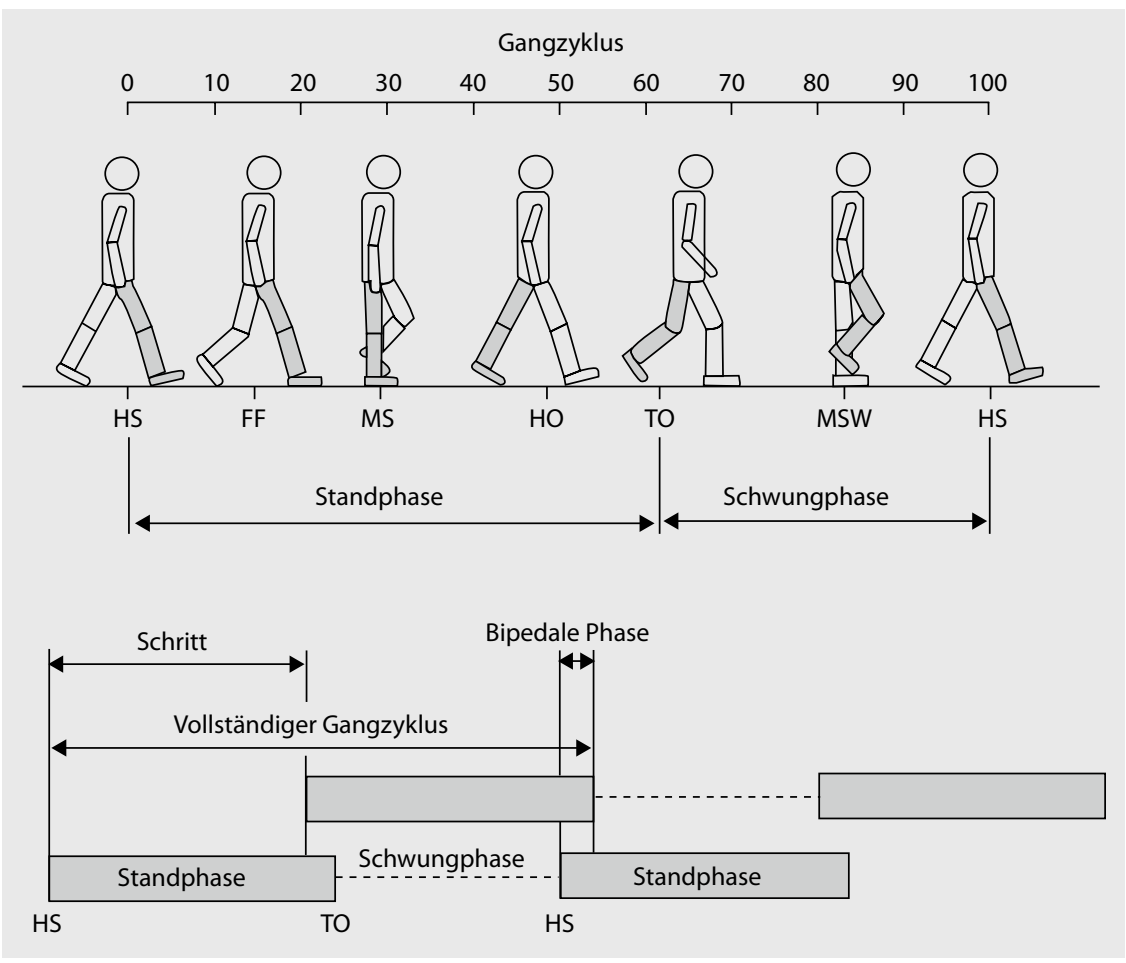

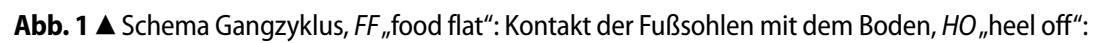

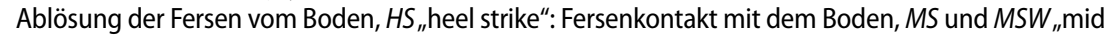
swing": mittlere Schwungphase, TO "toe off": Ablösung der Zehen vom Boden. (Aus [2]) 


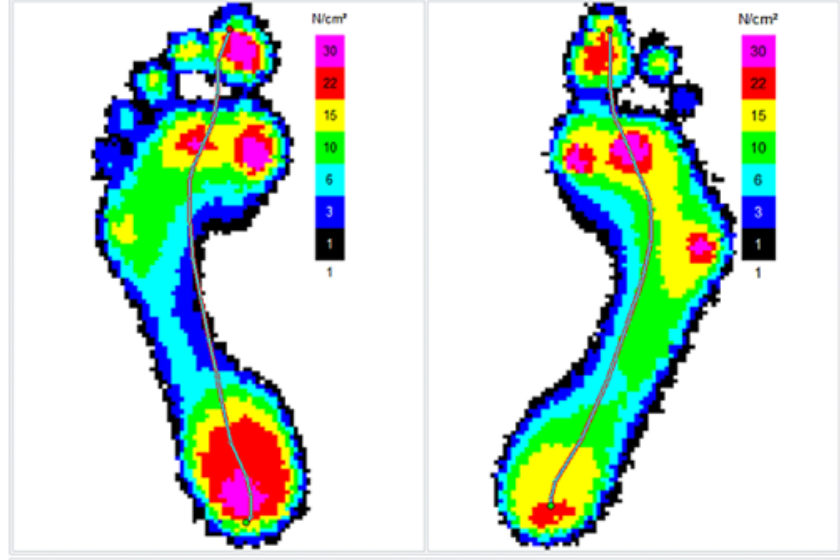

Abb. $2 \Delta$ Pedobarographie, physiologischer Befund des linken Fußes, Rückfußarthrodese des rechten Fußes mit verminderter Belastung der Ferse und Druckspitze auf den Kopf des Mittelfußknochens 5

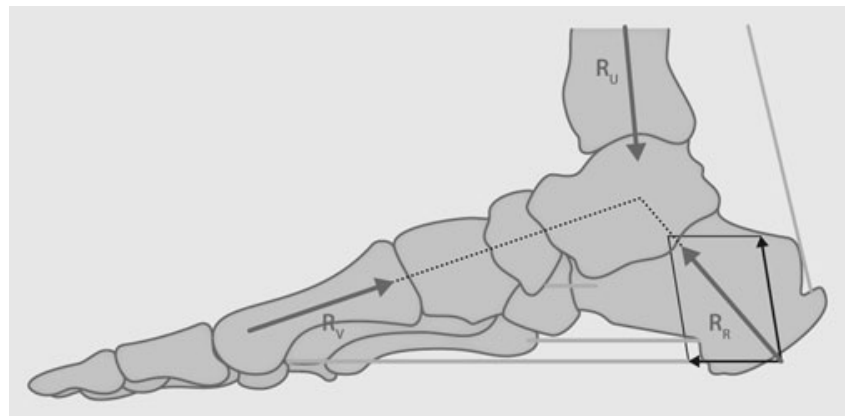

Abb. $4<$ Dreifachlängsverspannung des Fußes. (Nach [13])

verletzungen betroffen [8] - geschätzt gut 1 Mio. Mal pro Jahr in Deutschland. Die bandelastisch geführte Verankerung der Großzehe kann in der bekannten Hallux-valgus-Fehlstellung münden. Zudem ist der menschliche Fuß im Laufe des Lebens durch die hohe Belastung - geschätzte durchschnittliche Lebenslaufleistung 160 Mio. Schritte - und Alterungsprozesse gewissen Veränderungen unterworfen. So kommt es typischerweise zu einer Abflachung der Fußlängswölbung, einer Verringerung des plantaren Fettpolsters und der koordinativen Beweglichkeit.

\section{Gangzyklus}

Der Gang dient der Fortbewegung als Teil der Mobilität des modernen Menschen. Er ist zyklisch, wobei sich funktionell 2 Aufgaben differenzieren lassen:

- die Körpergewichtsübernahme in der Erstkontaktphase und

- die Verlagerung des Körpergewichts in der Stand- und Schwungphase nach vorne.

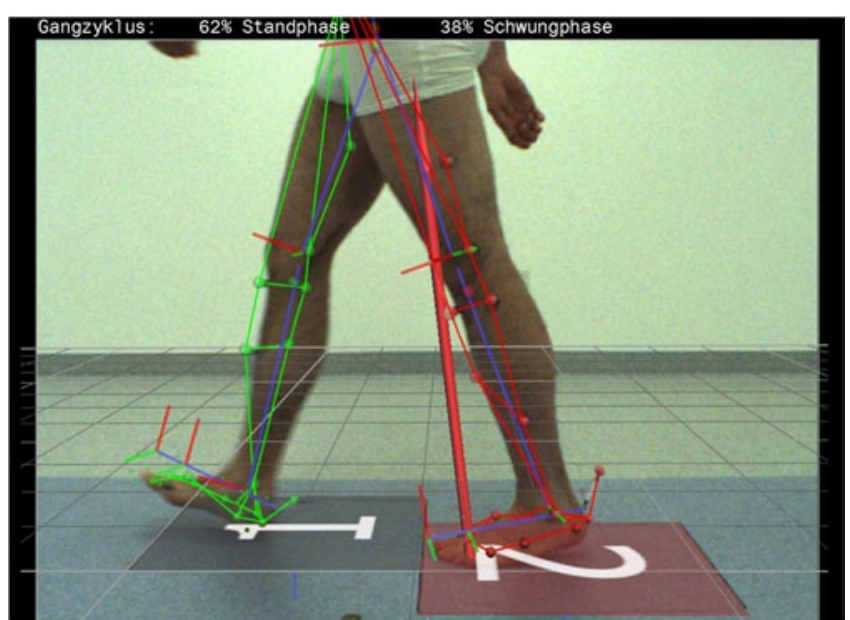

Abb. $3 \Delta$ Markerbasierte kinematische Ganganalyse im Ganglabor

im Bereich der Schuhorthopädietechnik und Einlagenversorgung [8].

Biomechanisch ist interessant, dass sich die Funktion des Fußes von einem sozusagen mobilen Adapter in der ersten Hälfte der Standphase (Stoßdämpferphase) zu einem quasi starren Hebel in der zweiten Hälfte (Abstoßphase, Propulsion) ändert ( $\bullet$ Abb. 3). Um dies zu ermöglichen, bedarf es einiger anatomisch-funktioneller Besonderheiten.

\section{Anatomische Aspekte}

\section{Knochen und Gelenke}

Der Fuß besteht aus 26 Knochen und regelhaft 2 Sesambeinen unter dem ersten Mittelfußkopf. Funktionell und anatomisch gesehen können diese Knochen in Gruppen zusammengefasst werden: Die anatomische Nomenklatur beschreibt

- den Tarsus mit den Fußwurzelknochen:

- Talus,

- Kalkaneus,

- Os naviculare (Kahnbein),

- Os cuboideum (Würfelbein) und

- den 3 Ossa cuneiformia (Keilbeine),

- den Metatarsus mit den 5 Mittelfußknochen und

- die Zehenknochen [11].

In der chirurgischen Praxis setzte sich die Einteilung in Rückfuß, Mittelfuß und Vorfuß durch:

Rückfuß. Er umfasst den Skelettabschnitt vom oberen Sprunggelenk (Talokruralge- 
lenk) bis zur Chopart-Gelenklinie (Articulatio tarsi transversa) und damit die beiden Knochen Talus und Kalkaneus.

Mittelfuß. Er besteht aus dem Os cuboideum und dem Os naviculare, den 3 Ossa cuneiformia mit der Lisfranc-Gelenklinie (Articulatio tarsometatarsale) und den Mittelfußknochen.

Vorfuß. Er beginnt an den Zehengrundgelenken (Articulatio metatarsophalangeae) und besteht aus den Fußzehen.

Der Fuß weist insgesamt 108 Gelenke auf, von welchen nicht alle für seine Funktion von gleich wichtiger Bedeutung sind. So lassen sich essenzielle von teilweise bzw. nichtessenziellen Fußgelenken unterscheiden $[4,9]$. Eine herausragende Stellung für eine physiologische Fußfunktion nehmen die 7 essenziellen Fußgelenke ein: OSG und Subtalargelenk, Talonavikulargelenk und die Zehengrundgelenke 2-5. Bei einer Störung der Mechanik und Beweglichkeit dieser Gelenke resultiert eine relevante Einschränkung der Funktion des gesamten Fußes. Dies ist bei der Behandlung von Gelenkverletzungen ebenso zu berücksichtigen, wie bei der Indikationsstellung für gelenkversteifende Eingriffe (Arthrodesen).

\section{Bänder und Muskulatur}

Da zum Gehen und Laufen eine Einheit mit funktionierender kinematischer Kette zwischen Unterschenkel und Fuß benötigt wird, sind die Knochen des Fußskeletts durch ein Netzwerk von insgesamt 57 Bändern über die Gelenke miteinander verbunden.

Besonders deutlich wird die Bedeutung eines intakten Bandapparats am oberen Sprunggelenk, welches seine federnde Festigkeit neben dem Außen- und Innenbandapparat v. a. durch die Syndesmose erhält.

Für die ligamentäre Führung und Stabilität des Fußes ist insbesondere das Lig. calcaneonaviculare plantare (Pfannenband, Springligament) zu nennen, das mit seinem Überzug aus Faserknorpel den Taluskopf im unteren Sprunggelenk abstützt. Zusammen mit dem Lig. plantare und wesentlich auch der Plantaraponeurose sorgt es außerdem für die Dreifachlängsverspannung der Fußlängswöl-

Trauma Berufskrankh 2015 - 17[Suppl 1]:4-9 DOI 10.1007/s10039-013-1991-0

(c) Springer-Verlag Berlin Heidelberg 2014

J. Gabel

\section{Funktionsanalyse des Fußes. Anatomische und biomechanische Aspekte für die chirurgische Praxis}

\section{Zusammenfassung}

Hintergrund. Der menschliche Fuß ist ein komplexes Körperorgan und das Resultat einer Millionen von Jahren dauernden Evolution, während welcher der Greif- und Kletterfuß zu einem menschlichen Lauf- und Standfuß umgebildet wurde. Die Biomechanik des Fußes ist geprägt von dieser funktionellen Anforderung und so anspruchsvoll wie faszinierend. Bemerkenswert ist, dass der moderne Mensch mit gesunden Füßen eine Gehleistung erreicht, die einer 2- bis 3-maligen Erdumrundung entspricht. Voraussetzungen für eine fußchirurgische Tätigkeit. Das Verständnis der Anatomie und Biomechanik des Fußes ist eine wesentli- che theoretische Grundlage für den in der Behandlung von Erkrankungen und Verletzungen des Fußes fußchirurgisch tätigen Orthopäden und Unfallchirurgen. Es wird ein praxisrelevanter Überblick über die wesentlichen biomechanischen Aspekte der Fußfunktion gegeben, womit das Interesse an diesem Thema geweckt werden soll. Für weitergehende Fragestellungen sei auf die entsprechende Fachliteratur verwiesen.

\section{Schlüsselwörter}

Fuß · Sprungbein · Fersenbein .

Pedobarographie · Metatarsalgie

\section{Functional analysis of the foot. Anatomic and biomechanical aspects for surgical practice}

\section{Abstract}

Background. The human foot is a complex organ and the result of a million year old evolutionary history. During this process, our feet evolved from grasping appendages into sturdy levers. Fascinating biomechanics are necessary for bipedal locomotion. It is exceptional that the modern human individual has a lifetime walking distance which is similar to 2-3 circumnavigations of the earth. Prerequisites for the foot surgery practice. Fundamental knowledge of anatomy and biomechanics are essential for the treatment of foot disorders and injuries. The following article explains relevant biomechanical aspects and generates interest for this topic.

\section{Keywords}

Foot · Talus · Calcaneus · Pedobarography . Metatarsalgia bung (- Abb. 4, [13, 14]). Diese längs ausgerichteten Bänder der Planta pedis sind Teil des passiven Stabilisierungssystems des Fußes und werden von aktiven Stabilisatoren synergistisch unterstützt. Dabei handelt es sich insbesondere um die langen Unterschenkelmuskeln, die in ihrem Verlauf das obere und untere Sprunggelenk überkreuzen. Damit steuern sie auch diese beiden Gelenke und sichern das dynamische Gleichgewicht dieses funktionellen Kardangelenks.

Für die aktive Unterstützung der Fußlängswölbung hat der M. tibialis posterior unter den extrinsischen Muskeln die wichtigste Funktion. Verständlich wird dies durch seinen Sehnenverlauf und Ansatz. Topographisch verläuft seine Sehne hinter dem Innenknöchel, unterfährt das Pfan- nenband und inseriert mit dem Hauptfaserzug an der Tuberositas ossis naviculare. Durch den Zug unter dem Pfannenband und seinen Ansatz an der medialen Fußsäule kontrolliert der M. tibialis posterior die Position des Taluskopfs [10]. Dessen Einstellung in eine physiologische talometatarsale Achse definiert die Konfiguration der Fußlängswölbung. Zusätzlich stabilisiert der M. tibialis posterior invertierend (Supination) den Rückfuß und ist Gegenspieler der Peronäalmuskulatur, die ebenfalls den Rückfuß umgreift und auf diesen evertierend (Pronation) wirkt. Dem M. peronaeus longus kommt zudem in Zusammenarbeit mit dem M. tibialis anterior eine stabilisierende Steigbügelfunktion zur Verspannung der Fußquerwölbung auf Höhe der Lisfranc-Gelenkli- 


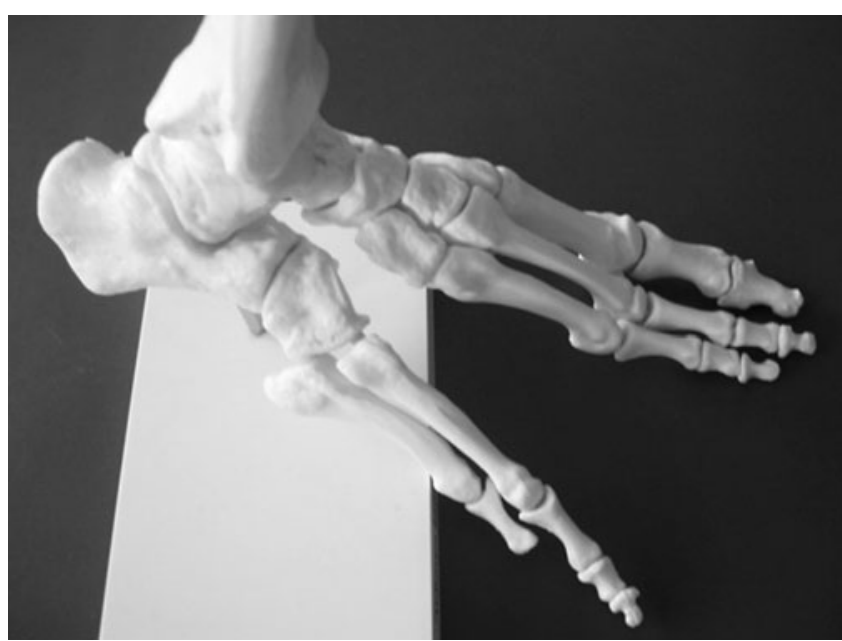

Abb. $5<$ SprungbeinFersenbein-Fuß-Modell am Kunststoffknochen mit Sprungbeinfuß (Talus, Os naviculare, Ossa cuneiformia, Mittelfußknochen 1-3) und Fersenbeinfuß (Kalkaneus, Os cuboideum, Mittelfußknochen 4 und 5)
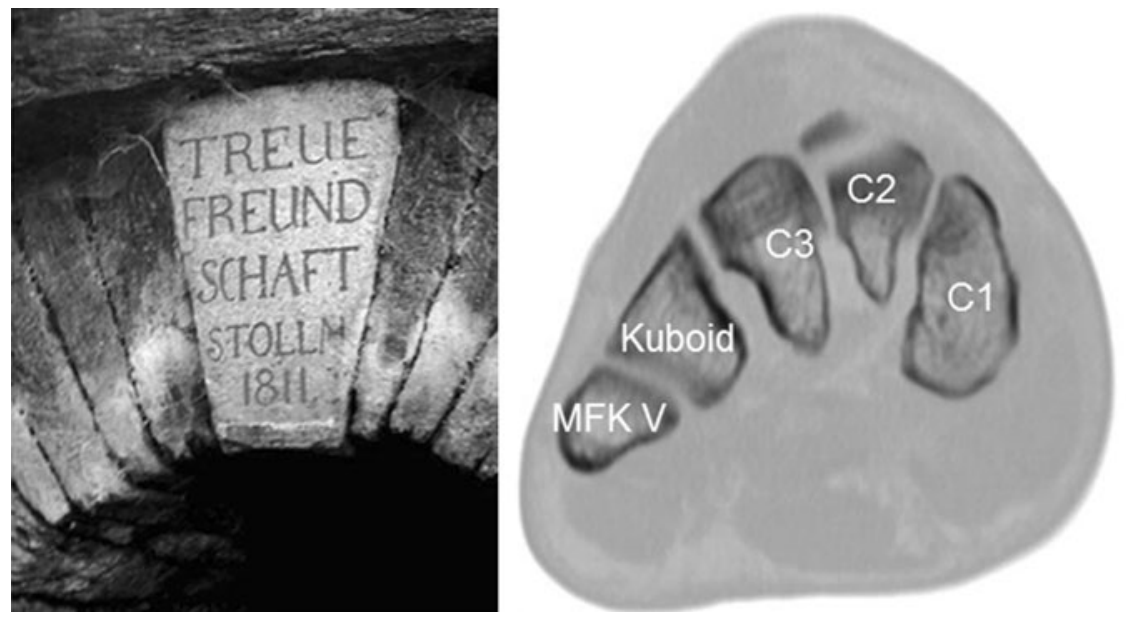

Abb. $7 \Delta$ Keilprinzip im tarsometatarsalen Gelenk, links zur Veranschaulichung, C1 Os cuneiforme mediale, C2 Os cuneiforme intermedium, C3 Os cuneiforme laterale, MFK Mittelfußknochen. (Mit freundl. Genehmigung von J. Gabel)

nie zu [12]. Auch die im Fuß liegende intrinsische Muskulatur ist Teil der aktiven Verspannung des Fußes.

\section{Zusammenwirken der aktiven und passiven Stabilisatoren}

Bei der dynamischen Stoßdämpfung des Fußes, wie sie beim Gehen, Laufen und beim Sprung erforderlich wird, wirken alle aktiven und passiven Stabilisierungssysteme des Fußes zusammen und sind in der Lage, das Vielfache des Körpergewichts gegen die Bodenreaktionskräfte aufzufangen, ohne dass knöcherne oder ligamentäre Schäden resultieren, was entsprechende Anforderungen an die Konstruktion stellt.

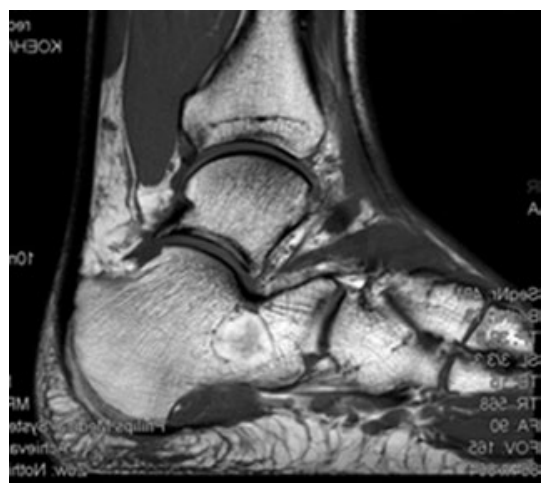

Abb. $6 \Delta$ Septiert gekammertes Fußfettgewebe und Trajektorenmuster der Substantia spongiosa des Kalkaneus

die ausgeklügelte Gewölbekonstruktion und Dämpfungsfunktion des Fußes [13]. Die wesentliche Dämpfung und Absorption von einwirkenden Kräften finden in den elastischen Komponenten der Fußmuskulatur und dem septiert gekammerten Fettpolster der Fußsohle statt, das hohen Flächendrücken standhalten kann (• Abb.6).

Die Gewölbekonstruktion ist nach dem sich selbst tragenden Keilprinzip aufgebaut, im Bereich der tarsometatarsalen Gelenklinie (Lisfranc-Gelenk) lässt die knöcherne Anatomie einen Quergewölbebogen erkennen, in dessen Scheitelpunkt sich die 3 Keilbeine (Ossa cuneiformia) befinden. Dies steht in Analogie zu einem steinernen Rundbogen, der durch einen Schlussstein seine nachhaltige Stabilität erhält (• Abb. 7).

Zur knöchernen Anatomie kommt das Spiralprinzip durch die aktiv einwirkende Fußmuskulatur hinzu. Dabei üben der M. peronaeus longus einen pronierenden und der M. tibialis posterior einen supinierenden Effekt aus. Aus den gegensinnig wirkenden Kräften resultieren eine Verwindung und dynamische Stabilisierung der Quergewölbekonstruktion.

Das Längsgewölbe wird dynamisch durch den Windlass-Mechanismus stabilisiert (• Abb. 8). Im Ingenieurwesen ist dies die englische Bezeichnung für eine Ankerwinde, die aus einem horizontalen Zylinder besteht, über den ein Seil gezogen wird. Auf den Fuß übertragen wird durch die Dorsiflexion der Großzehe über den Mittelfußkopf des ersten Strahls (Zylinder der Winde) die Plantaraponeurose (Seil) angespannt. Durch diesen Mecha- 


\section{Funktionsstörungen des Fußes}

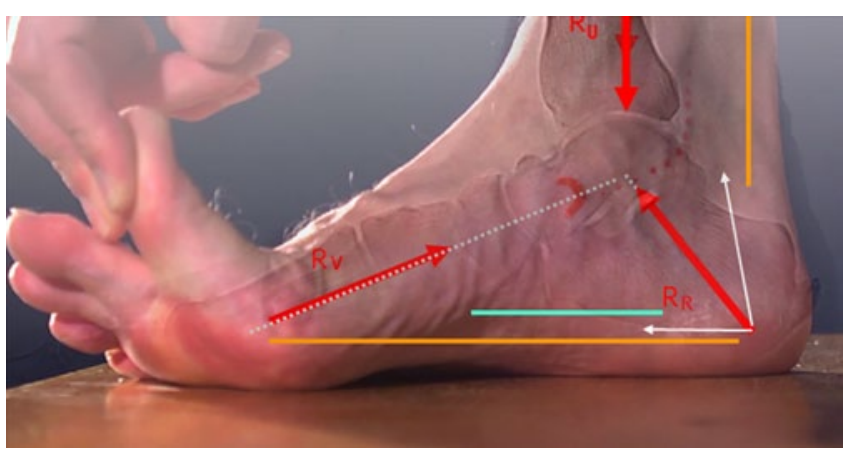

Abb. 8 \ Windlass-Mechanismus - Verspannung der Fußlängswölbung

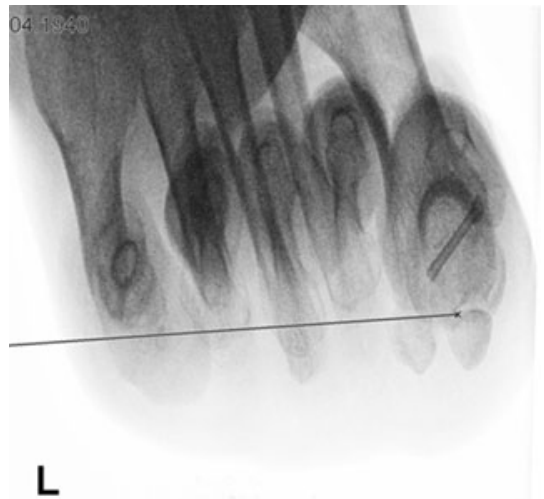

Abb. $10 \Delta$ Sprinteraufnahme mit Nachweis einer relativen Elevatusstellung der Mittelfußknochen 2, 4 und 5 als radiologisches Korrelat einer klinisch diagnostizierten Metatarsalgie unter dem 2. Mittelfußkopf nach verkürzender Weil-Osteotomie der Mittelfußknochen

nismus ist der Fuß in der Lage, einen großen Anteil der Belastung auf die Plantaraponeurose zu übertragen, in der während eines Gangzyklus Zugkräfte von bis zu 50\% des Körpergewichts auftreten können, während sie im Stehen kaum belastet wird [12]. Außerdem wird durch diese Verspannung eine Überlastung der Mittelfußknochen durch Biegekräfte verhindert. Für den ersten Strahl resultiert damit eine axiale Belastung. Bei einem ausgeprägten Hallux valgus ist dieser Mechanismus empfindlich gestört, die Fußlängswölbung verliert ihre wichtigste Verspannung und flacht sich ab.

Ein anderer Mechanismus ist für die Fußfunktion im Abrollvorgang von zentraler Bedeutung: der sog. Verblockungsmechanismus („locking mechanism“) im Chopart-Gelenk (Articulatio tarsi transversa). Dieses Gelenk ist nicht nur das für die Pro- und Supination entscheidende

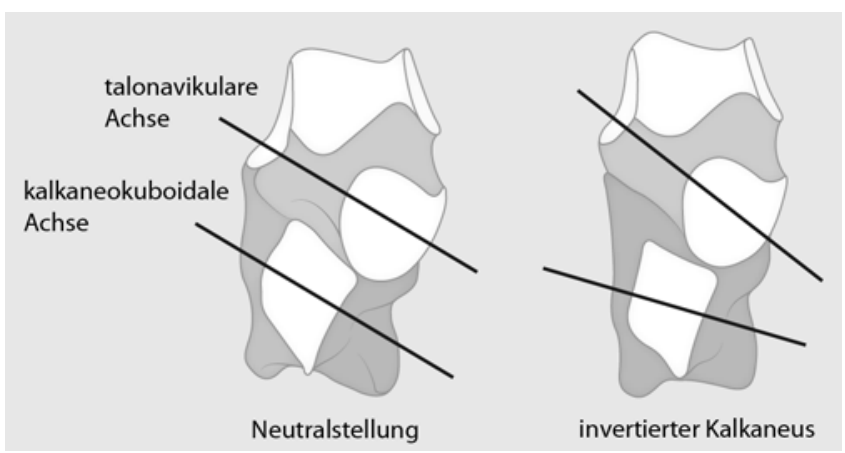

Abb. $9 \Delta$ Achsausrichtung der Gelenke im Chopart-Gelenk in Neutralstellung: freie Beweglichkeit durch parallele Achsausrichtung, Gelenkblockade bei varischer Rückfußeinstellung

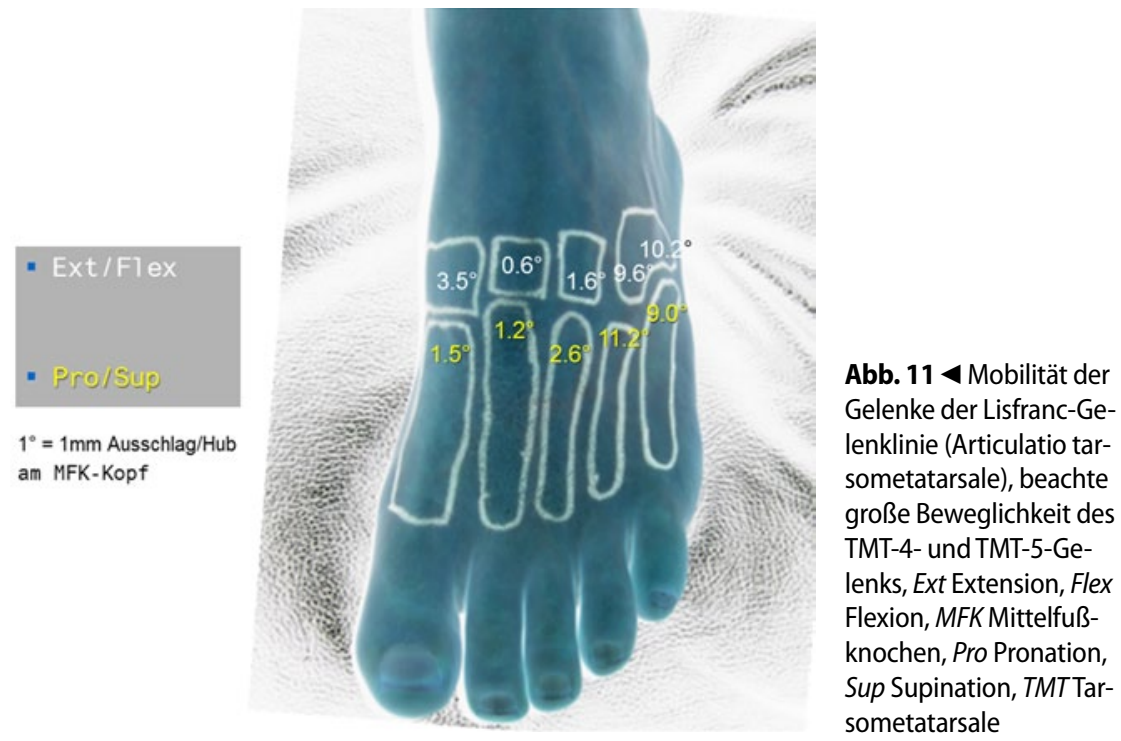

Fußgelenk, sondern besitzt auch die biomechanische Eigenschaft, bei pronatorischer Verdrehung in eine bandgebremste Blockade geführt zu werden [3]. Möglich wird dies durch die Morphologie und die Anatomie der beteiligten Gelenke: Talonavikular- und Kalkaneokuboidgelenk, deren Achsen nur in Neutral-, nicht jedoch in Pronationsstellung parallel ausgerichtet sind (schematisch in $\square$ Abb. 9 dargestellt).

Aktiv wird die Verblockung durch den pronierenden Sehnenzug des M. tibialis posterior gesteuert. Dadurch wird erst die Übertragung der über den Rückfuß wirkenden Kräfte der Achillessehne auf den Mittel- und Vorfuß ermöglicht. Resultat sind eine physiologische Propulsion des Fußes im Abrollvorgang und die Fähigkeit zum Zehenspitzengang.

\section{Praktische Umsetzung}

Für die klinische Praxis lässt sich ableiten, dass bei Durchführung von Arthrodesen in den Sprunggelenken eine neutrale bis leicht valgische Einstellung des Rückfußes im Subtalar- und Chopart-Gelenk zum Erhalt der Vor- und Mittelfußbeweglichkeit unbedingt anzustreben ist. Denn bei varischer Einstellung des Rückfußes ist der Fuß ähnlich einem Ballenhohlfuß (Pes cavovarus) verblockt und wird unphysiologisch am Fußaußenrand überlastet.

Der Ausfall des oben erläuterten sog. Verblockungsmechanismus und die fehlende Stabilisierung der Fußlängswölbung zeigen sich anschaulich am Krankheitsbild der Tibialis-posterior-Dysfunktion, dessen höchste Inzidenz sich bei Frauen im Alter über 40 Jahren findet. Ursächlich ist eine chronische Sehnenruptur auf 
Basis einer Texturstörung, welche meist mit einer Ruptur bzw. Degeneration des Lig. calcaneonaviculare plantare (Pfannenband) einhergeht. Progredient führt dies zu einer statischen Veränderung und zunehmenden Deformität des Fußes mit schmerzhaftem Pes planovalgus [3].

Bezüglich der Ausbildung der Fußquerwölbung ist heute anerkannt, dass sie anatomisch und biomechanisch nur im Bereich des Mittelfußes angelegt ist. Das Vorfußquergewölbe, das man in der klinischen Untersuchung am entlasteten Fuß erkennt, flacht sich unter Belastung vollständig ab $[1,12]$. Auch dieser Mechanismus ist ein Element der Stoßdämpferfunktion des Fußes. Die pedobarographische Untersuchung zeigt einen zusammenhängenden Druckkontakt unter den Mittelfußköpfen am dynamisch belasteten Fuß. Die früher diskutierte Dreipunktauflage des Fußes lässt sich also nicht bestätigen und wäre aus statischen Überlegungen auch nachteilig, da die bereits kleine Standfläche des Fußes von etwa $100 \mathrm{~cm}^{2}$ noch mehr reduziert wäre. Bei einem gesunden Fuß werden gerade die zentralen Mittelfußköpfe 2 und 3 mit einem hohen Anteil der Lastübernahme in der Abstoßphase (Propulsion) belastet.

Bei einer Störung des Alignments der Mittelfußköpfe ist eine schmerzfreie Belastung der Zehenballen im Abrollvorgang meist nicht mehr möglich. Diese als Metatarsalgie bezeichnete Vorfußpathologie wird in ihren verschiedenen Formen in einer Fußsprechstunde häufig diagnostiziert und ist beispielsweise im Sinne einer Transfermetatarsalgie durch reduzierte Lastübernahme des 1 . Strahls beim symptomatischen Hallux valgus unter dem 2. und 3. Mittelfußkopf problematisch. Auch eine ausgeprägte Spreizfußkomponente kann die Ursache für derartige Schmerzen im Vorfuß sein.

Die Untersuchung der Druckverteilung beinhaltet die Beurteilung der Fußsohle auf mögliche pathologische Druckschwielen und kann mit einer Pedobarographie sowie einer Spezialröntgenaufnahme, der sog. Sprinteraufnahme, ergänzt werden (• Abb. 10).

Posttraumatisch kann bei Luxationsverletzungen des Lisfranc-Gelenks oder dislozierten Frakturen des Mittelfußes durch oft unterschätzte Achsabweichun- gen der Mittelfußknochen ein biomechanisch relevantes Metatarsalemalalignment verbleiben. Dies muss in der Primärversorgung des unfallverletzten Fußes beachtet werden, da aufgrund der Länge der Mittelfußknochen bereits eine minimale Achsabweichung von $1^{\circ}$ in den Mittelfußgelenken (Articulatio tarsometatarsale) oder basisnah zu einer Elevatusfehlstellung bzw. Plantarisierung des entsprechenden Mittelfußkopfs von $1 \mathrm{~mm}$ führt. Diese Abweichung ist insbesondere für die relativ straff in den Mittelfußgelenken 2 und 3 geführten zentralen Mittelfußknochen (Amphiarthrosen) schlecht zu kompensieren.

Für die laterale Fußsäule ist zu beachten, dass die große Mobilität der Mittelfußknochen 4 und 5 als Teil ihrer Flexibilität erhalten werden muss. Arthrodesen der betreffenden Gelenke, deren Beweglichkeit in $\bullet$ Abb. 11 dargestellt ist, sollten vermieden werden. Gleiches gilt für die Grundgelenke der Kleinzehen, da diese essenzielle Fußgelenke sind. Eine gut eingestellte Arthrodese des Großzehengrundgelenks dagegen kann gut kompensier werden und geht in der Regel nicht mit wesentlichen biomechanischen Einschränkungen einher.

\section{Fazit für die Praxis}

Fußprobleme des modernen, zivilisatorisch lebenden Menschen sind sehr häufig, jeder dritte Erwachsene klagt darüber [13].

- Die Kenntnis der Grundzüge der Biomechanik des Fußes ist die Basis für die konservative und operative Therapie von Pathologien am krankhaft veränderten und unfallverletzten Fuß.

Nicht nur der auf den Fuß spezialisierte Unfallchirurg und Orthopäde, sondern jeder chirurgisch Tätige sollte über das notwendige Basiswissen bezüglich der Biomechanik des Fußes verfügen.

\section{Korrespondenzadresse}

\section{J. Gabel}

Abteilung für Fußchirurgie, BG-Unfallklinik Murnau,

Prof.-Küntscher-Straße 8, 82148 Murnau jgabel@bgu-murnau.de

\section{Einhaltung ethischer Richtlinien}

Interessenkonflikt. J. Gabel gibt an, dass kein Interessenskonflikt besteht.

Dieser Beitrag beinhaltet keine Studien an Menschen oder Tieren.

The supplement containing this article is not sponsored by industry.

\section{Literatur}

1. Andermahr J, Jubel A, Rehm KE, Koebke J (2011) Erkrankungen und Verletzungen des Rückfußes. Deutscher Ärzteverlag, Köln

2. Beckers D, Deckers J (1997) Ganganalyse und Gangschulung. Therapeutische Strategien für die Praxis. Springer, Berlin Heidelberg New York

3. DeSilva JM (2013) The lower limb and mechanism of walking in Australopithecus sediba. Sience 340:6129

4. DiGiovanni $\mathrm{CH}$, Benirschke $S$, Hansen ST (2003) Foot injuries. In: Browner B, Jupiter J, Levine A, Trafton P (Hrsg) Skeletal trauma. Saunders, Philadelphia, Chap 60, S 2375-2377

5. Götz-Neumann K (2003) Gehen verstehen - Ganganalyse in der Physiotherapie. Thieme, Stuttgart New York

6. Hayafune N, Hayafune Y (1999) Pressure and force distribution characteristics under the normal foot during the push-off phase in gait. Foot 9:88-92

7. Kummer B (2005) Biomechanik - Form und Funktion des Bewegungsapparates. Deutscher Ärzteverlag, Köln

8. Larsen C (2007) Gut zu Fuß ein Leben lang. Trias, Stuttgart

9. Niethard FU (2009) Orthopädie und Unfallchirurgie. Duale Reihe. Thieme, Stuttgart New York

10. Ouzounian TJ, Shereff MJ (1989) In vitro determination of midfoot motion. Foot Ankle 10:140-146

11. Perry J (1992) Ganganalyse - Norm und Pathologie des Gehens. Urban \& Fischer, München

12. Schad W (2000) Der Fuß des Menschen - ein lange verkanntes Detail seiner Evolution. DAF News 5(2):S10-S13

13. Tillmann B (1977) Beitrag zur funktionellen Anatomie des Fußes. Orthop Prax 13:505-509

14. Valderrabano V, Engelhardt M, Küster HH (2009) Fuß \& Sprunggelenk und Sport. Deutscher Ärzteverlag, Köln 